\title{
Prontuário eletrônico do paciente: qual a contribuição da arquivística e do Smart Contracts para a sua gestão na Era da Saúde 4.0?
}

\section{Electronic health records: what is the contribution of archivistics and Smart Contracts for their management during the period of Health 4.0?}

\author{
Andréia Castro Costa Xavier ${ }^{1}$, Cláudio Gottschalg-Duque ${ }^{2}$ \\ ${ }^{1}$ Universidade de Brasília (UnB), Brasília, DF, Brasil. ORCID: https://orcid.org/0000-0003-2714-4058 \\ 2 Universidade de Brasília (UnB), Brasília, DF, Brasil. ORCID: https://orcid.org/0000-0003-3558-466X
}

Autor para correspondência/Mail to: Andréia Castro Costa Xavier, andreiaccx@gmail.com

Recebido/Submitted: 30 de maio de 2021; Aceito/Approved: 01 de julho de 2021

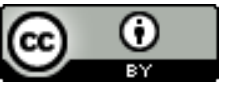

Copyright (C) 2021 Xavier \& Gottschalg-Duque. Todo o conteúdo da Revista (incluindo-se instruções, política editorial e modelos) está sob uma licença Creative Commons Atribuição 4.0 Internacional. Ao serem publicados por esta Revista, os artigos são de livre uso em ambientes educacionais, de pesquisa e não comerciais, com atribuição de autoria obrigatória. Mais informações em http://revistas.ufpr.br/atoz/about/submissions\#copyrightNotice.

\begin{abstract}
Resumo
Introdução: O prontuário eletrônico do paciente constitui a base de apoio para os avanços tecnológicos na área de Saúde 4.0, expressão alusiva à Quarta Revolução Industrial ou Indústria 4.0. As inovações nesta área contemplam o emprego de robótica, inteligência artificial e conectividade para a prestação de serviços ao paciente. Esta pesquisa teve como pressuposto o fato de que o emprego de métodos arquivísticos e das tecnologias Blockchain e Smart Contracts pode contribuir positivamente para a gestão dos prontuários eletrônicos do paciente. Os aspectos contemplados por esta gestão se referem à produção, ao uso e à destinação dos prontuários, bem como à proteção, segurança e privacidade dos seus dados. Método: A busca sistemática da literatura e os estudos das obras da subárea "Arquivologia" foram os métodos utilizados para identificar e descrever as atividades de uma gestão arquivística de documentos, os atributos das tecnologias Blockchain e Smart Contracts e a relação entre estes temas com a gestão dos prontuários. A pesquisa teve um caráter exploratório, descritivo, qualitativo e uma perspectiva ontológica construtivista. Resultado: Os estudos contribuíram para constatar lacunas na legislação e na produção de artigos científicos sobre o tema investigado, bem como para identificar possíveis soluções tecnológicas para resolução de questões técnicas até então não elucidadas. Conclusão: A participação dos arquivistas em pesquisas relativas à gestão arquivística de documentos associada às tecnologias Blockchain e Smart Contracts é necessária para contribuir para a evolução da gestão de documentos da qual os prontuários eletrônicos do paciente fazem parte.
\end{abstract}

Palavras-chave: Prontuário eletrônico do paciente; Arquivística; Blockchain; Smart Contracts; Saúde 4.0.

${ }^{a}$ Segundo Araújo (2018), a Arquivologia é uma subárea da Ciência da Informação.

\begin{abstract}
Introduction: The electronic health record constitutes a support base for technological advances in the Health 4.0 area, an expression alluding to the Fourth Industrial Revolution or Industry 4.0. The innovations in this area include the use of robotics, artificial intelligence and connectivity to provide services to the patient. This research was presupposed on the fact that the use of archivistics methods and Blockchain and Smart Contracts technologies can contribute positively to the management of the patient's electronic health records. The aspects covered by this management are defined in the production, use and destination of health records as well as the protection, security and privacy of your data. Method: The systematic search of the literature and studies of the works in the sub-area "Archivology" were the methods used to identify and describe as activities of an archivistics management, the attributes of the technologies Blockchain and Smart Contracts and the relationship between these themes with the governance of health records. The research had an exploratory, descriptive, qualitative character and a constructivist ontological perspective. Result: The studies contributed to finding gaps in the legislation and in the production of scientific articles on the subject investigated, as well as to identify possible technological solutions to problem-solving of technical issues that had not yet been clarified. Conclusion: The participation of archivists in research related to archivistics management associated with Blockchain and Smart Contracts technologies is necessary to contribute to the evolution of record management, of which the patient's electronic health records are part.
\end{abstract}

Keywords: Eletronic health records; Archivistics; Blockchain; Smart Contracts; Health 4.0.

\section{INTRODUÇÃO}

Esta pesquisa apresenta as contribuições da gestão arquivística de documentos ${ }^{1}$ e das tecnologias Blockchain e Smart Contracts para a gestão e segurança do Prontuário Eletrônico do Paciente (PEP). Além disso, objetiva descrever os conceitos e os principais componentes de uma gestão arquivística para os PEPs; identificar as características e o funcionamento das tecnologias Blockchain e Smart Contracts; e demonstrar as vantagens de uma gestão integrada dos PEPs por meio da aplicação das técnicas arquivísticas e das tecnologias Blockchain e Smart Contracts como contribuição à Saúde 4.0 .

\footnotetext{
${ }^{1}$ Conforme Jardim (2018, p. 40), "gestão arquivística refere-se [...] ao conjunto de elementos mobilizados para a gerência de serviços e instituições arquivísticas".
} 
O PEP é considerado um documento arquivístico conceituado como "documento produzido (elaborado ou recebido), no curso de uma atividade prática, como instrumento ou resultado de tal atividade, e retido para ação ou referência." (Conselho Nacional de Arquivos, 2020a, p. 24). Por ser um documento arquivístico, este deve fazer parte de uma gestão de documentos ${ }^{2}$ definida como "o conjunto de procedimentos e operações técnicas referentes à sua produção, tramitação, uso, avaliação e arquivamento em fase corrente e intermediária, visando a sua eliminação ou recolhimento para guarda permanente."(Lei no 8.159, de 8 de janeiro de 1991).

Referido PEP é utilizado para registro e acesso às informações sobre o paciente, motivo pelo qual são necessárias ações de governança ${ }^{3}$ para a eficiência na sua gestão. Tais ações contemplam desde a definição de seus metadados, layout, classificação arquivística, acesso, recuperação, armazenamento até a sua preservação digital. Toda esta curadoria requer orientação normativa, utilização de métodos e tecnologias adequados e capacitação de pessoal. O Conselho Federal de Medicina atua para tornar a gestão eficiente do PEP definido:

como o documento único constituído de um conjunto de informações, sinais e imagens registradas, geradas a partir de fatos, acontecimentos e situações sobre a saúde do paciente e a assistência a ele prestada, de caráter legal, sigiloso e científico, que possibilita a comunicação entre membros da equipe multiprofissional e a continuidade da assistência prestada ao indivíduo (Resolução CFM n. 1.638, de 10 de julho de 2002).

A Indústria 4.0 citada na Feira Hannover, em 2011, se refere à nova geração de tecnologias que, de acordo com Nabeto (2020, p. 7) "está a funcionar de forma integrada, ligando o espaço virtual ao mundo físico". Estas tecnologias avançam na área de saúde, denominada Saúde 4.0, com potenciais para tornar os serviços "inteligentes", por meio do emprego de inteligência artificial e robótica, otimizar o trabalho dos profissionais de saúde e elevar os níveis de satisfação dos pacientes. O PEP é o documento chave para apoiar a evolução desses serviços de saúde e necessita de uma governança alicerçada na gestão arquivística e tecnológica de seus dados.

A gestão arquivística de documentos contempla uma política arquivística, responsáveis e um programa de gestão documental. A política contém orientações necessárias para a produção, manutenção e preservação de documentos com as características de confiabilidade, autenticidade, acessibilidade e legibilidade para apoiar as funções e atividades organizacionais. A designação de responsáveis tem como intuito reunir profissionais para coordenar, supervisionar, fomentar e fortalecer a gestão de documentos. Além disso, é composta por quatro instrumentos principais e três adicionais de trabalho. O plano de classificação de documentos ${ }^{4}$ (PCD), a tabela de temporalidade e destinação de documentos (TTDD), o manual de gestão arquivística de documentos e o esquema de classificação referente à segurança e ao acesso aos documentos correspondem aos instrumentos principais. $\mathrm{O}$ glossário, o vocabulário controlado e o tesauro constituem os instrumentos adicionais. Um Sistema informatizado de gestão arquivística de documentos (SIGAD) é necessário para apoiar todas as fases da gestão documental, além das técnicas de preservação e segurança da informação (Conselho Nacional de Arquivos, 2020b, p.32-34).

A gestão tecnológica dos PEPs contempla o emprego da tecnologia Blockchain considerada disruptiva e inovadora em termos de segurança e descentralização da gestão de dados acessíveis, transacionados e trafegados pela Internet. Drescher (2018, p. 7) esclarece "Essa ferramenta apresenta-se como uma rede distribuída que funciona em blocos, onde as informações registradas são criptografadas, garantindo assim a inalterabilidade, privacidade e segurança."Na Era da Sociedade em Rede Castells (2013), os PEPs estão sujeitos aos perigos decorrentes de ataques cibernéticos, colocando as informações pessoais neles contidas sob forte necessidade de proteção contra acessos indevidos e adulterações, o que poderá ser viabilizado por meio do uso desta tecnologia.

Satoshi Nakamoto ${ }^{5}$ é o pseudônimo do criador da tecnologia Blockchain cuja finalidade inicial, ao ser criada em 2008, era permitir a realização de pagamentos em moedas digitais (criptomoedas) entre duas ou mais pessoas, sem intervenção bancária ou governamental, sendo que a Bitcoin foi a primeira moeda utilizada. As características diferenciadas e promissoras desta tecnologia fizeram com que o seu uso se expandisse para além das criptomoedas e, atualmente, a sua utilização se difundiu para diversos segmentos tais como logística, seguros, saúde, pagamentos governamentais, eleições e smart contracts, com a finalidade de propiciar rapidez, independência e segurança nas transações realizadas por meio de suas redes, sem a intermediação de terceiros como bancos e cartórios.

Os Smart Contracts, também chamados de Contratos Inteligentes Ethereum (2021), são uma outra tecnologia, porém, os desenvolvedores criaram seus programas computacionais autoexecutáveis na plataforma Blockchain devido aos atributos e benefícios oferecidos por esta tecnologia. A vantagem dos Smart Contracts em comparação com a Blockchain é que estes são flexíveis e permitem a programação dos sistemas de negócio de acordo com as suas necessidades e requisitos, diferentemente da Blockchain, que não oferece esta possibilidade. Segundo Ferraz (2019, p. 48), Smart Contracts é "um programa de computador, escrito em linguagem computacional que uma determinada máquina pode interpretar e executar". Um sistema dessa natureza pode ser programado para executar atividades tais como registro, validação e permissão de acesso ao usuário, em uma conta na blockchain,

\footnotetext{
${ }^{2}$ A Lei $\mathrm{n}^{\circ}$ 8.159, de 8 de janeiro de 1991, dispõe sobre a política nacional de arquivos públicos e privados.

${ }^{3}$ Para Gonçalves (2006), "Governança diz respeito aos meios e processos que são utilizados para produzir resultados eficazes".

${ }^{4}$ Quando um Plano de classificação de documentos recebe códigos numéricos ou alfanuméricos para designar as classes, subclasses e grupos denomina-se Código de classificação de documentos.

${ }^{5}$ Autor do artigo "Bitcoin: A Peer-to-Peer Eletronic Cash System".
} 
para gerenciar as permissões de acesso relacionadas aos PEPs Viana, Brandão, Dias, Castellano, e Guimarães (2020). Este programa computacional poderá auxiliar a governança dos PEPs tanto em termos de segurança e privacidade, com a proteção de seus dados por meio de criptografia ${ }^{6}$, quanto de automação de atividades, com comandos autoexecutáveis.

\section{A Gestão arquivística e tecnológica dos PEPs}

Uma governança eficiente dos PEPs depende de uma Arquitetura da Informação (AI) que combine a sua gestão arquivística com o uso de tecnologia. Para Vidotti, Cusin, e Corradi (2008, p. 182):

Arquitetura da informação enfoca a organização de conteúdos informacionais e as formas de armazenamento e preservação (sistemas de organização), representação, descrição e classificação (sistema de rotulagem, metadados, tesauro e vocabulário controlado), recuperação (sistema de busca), objetivando a criação de um sistema de interação (sistema de navegação) no qual o usuário deve interagir facilmente (usabilidade) com autonomia no acesso e uso do controle (acessibilidade) no ambiente hipermídia informacional digital.

A estruturação da gestão arquivística dos PEPs requer a realização de um diagnóstico e a elaboração de um projeto arquivístico, também chamado de projeto de trabalho. Segundo Lopes (2009, p. 159), "o projeto de trabalho é um instrumento de planejamento das atividades, concebido como decorrência dos problemas diagnosticados". Pelo que se pôde inferir a CTDE/Conarq (2020b, p.34) substituiu o uso dos termos diagnóstico ${ }^{7}$ e projeto pela atividade denominada de Planejamento do Programa de Gestão Arquivística, que se encontra citada na publicação e-Arq Brasil, como demonstrado a seguir:

o planejamento envolve o levantamento e a análise da realidade institucional, o estabelecimento das diretrizes e procedimentos a serem cumpridos pelo órgão ou entidade, o desenho do sistema de gestão arquivística de documentos e a elaboração de instrumentos e manuais (Conselho Nacional de Arquivos, 2020b, p. 34).

Os PEPs integrantes de um programa arquivístico de documentos serão classificados de acordo com um plano de classificação e comporão uma tabela de temporalidade criados para permitir a sua recuperação e posterior cumprimento de prazos de guarda e destinação para eliminação ou guarda permanente. O PCD "É um esquema de distribuição de documentos em classes, de acordo com métodos de arquivamento específicos, elaborado a partir do estudo das estruturas e funções de uma instituição e da análise do arquivo por ela produzido"(Conselho Nacional de Arquivos, 2020a, p. 39). Os prazos de guarda, instituídos aos PEPs pela TTDD, possuem como finalidade:

conservar os documentos necessários ao cumprimento de obrigações legais e de prestação de contas; conservar os documentos importantes para a memória corporativa; eliminar os documentos que não são mais necessários; e atender às necessidades e interesses de pessoas ou instituições externas ao órgão ou entidade [...] (Conselho Nacional de Arquivos, 2020b, p. 59-60).

Há uma diferença no estabelecimento de prazos de guarda e destinação de PEPs em geral e de servidores públicos. Para os primeiros a guarda é de, no mínimo, 20 anos, a partir do último registro inscrito no prontuário, e a destinação é eliminação, exceto para documentos identificados como de valor histórico pela Comissão Permanente de Revisão de Prontuários e Avaliação de Documentos ${ }^{8}$. O prazo de guarda ${ }^{9}$ dos PEPs de servidores públicos é de 5 anos na fase corrente, 95 na fase intermediária e a destinação é a eliminação, independente do suporte, ou seja, papel ou meio digital. A eliminação é definida como a "destruição de documentos que, na avaliação, foram considerados sem valor para a guarda permanente, impedindo qualquer possibilidade de reconstrução"(Conselho Nacional de Arquivos, 2020a, p. 27). A eliminação de PEPs depende da aprovação de uma Comissão Permanente de Avaliação de Documentos $\left(\mathrm{CPAD}^{10}\right)$, cujas atribuições podem ser exercidas pela Comissão de Permanente de Revisão de Prontuários e Avaliação de Documentos ${ }^{11}$

O Manual de gestão arquivística de documentos é um dos instrumentos de trabalho e contempla os procedimentos envolvendo produção, classificação, uso, avaliação e operação de sistemas arquivísticos, nos quais incluem-se os

\footnotetext{
${ }^{6}$ A "Criptografia é um método de codificação de documentos segundo um código secreto (chave), de modo que não possam ser apresentados de forma legível ou inteligível por uma aplicação e somente usuários autorizados sejam capazes de restabelecer sua forma original."(Conselho Nacional de Arquivos, 2020a, p. 113).

${ }^{7} \mathrm{O}$ termo diagnóstico "nem sempre é utilizado em outras línguas"(Braga \& Roncaglio, 2019, p. 390).

${ }^{8}$ A Lei n. 13.787, de 27 de dezembro de 2018, dispõe sobre a digitalização e a utilização de sistemas informatizados para a guarda, o armazenamento e o manuseio de prontuário de paciente e sobre a Comissão Permanente de Revisão de Prontuários e Avaliação de Documentos.

${ }^{9}$ A Portaria AN n ${ }^{\circ} 47$, de 14 de fevereiro de 2020, dispõe sobre o Código de Classificação e Tabela de Temporalidade e Destinação de Documentos relativos às atividades-meio do Poder Executivo Federal.

${ }^{10}$ O Decreto n. 10.148, de 2 de dezembro de 2019, institui as Comissões Permanentes de Avaliação de Documentos, no âmbito dos órgãos e das entidades da administração pública federal, órgãos técnicos com o objetivo de orientar e realizar o processo de análise, avaliação e seleção dos documentos produzidos e acumulados no seu âmbito de atuação.

${ }^{11}$ A Resolução CFM no 1.638 , de 10 de julho de 2002, define prontuário médico e torna obrigatória a criação da Comissão de Revisão de Prontuários nas instituições de saúde.
} 
PEPs Conselho Nacional de Arquivos (2020b). O esquema de classificação de acesso, proteção e segurança dos PEPs poderá fazer parte deste manual, uma vez que também compõe os instrumentos de gestão. O SIGAD é outro instrumento de trabalho recomendado para apoiar a gestão arquivística de documentos cujo modelo de requisitos é conhecido como e-ARQ Brasil ${ }^{12}$. O Conselho Nacional de Arquivos (Conarq) recomenda a adoção das Diretrizes para a implementação de Repositórios Digitais Confiáveis de Documentos Arquivísticos ${ }^{13}$ para a garantia da integridade, autenticidade, confidencialidade, disponibilidade e da preservação de documentos, os quais se incluem os PEPs.

A diversidade de padrões e protocolos utilizados pelas organizações de saúde acarretam dificuldades para a interoperabilidade e o compartilhamento de informações sobre os pacientes. Porém, tais dificuldades podem ser solucionadas por meio da disponibilização dessas informações em padrões abertos e com regulamentações baseadas na tecnologia Blockchain, com a curadoria das regras de negócio executadas pelo Smart Contracts. Estas tecnologias contribuirão para o compartilhamento de dados dos prontuários entre organizações de saúde, bem como para conceder ao paciente o direito de escolher com quais instituições compartilhar seus dados de acordo com os critérios de privacidade, auditabilidade e confiança Mayer, da Costa, e Righi (2020).

A Blockchain possui um legder, uma espécie de livro razão digital, similar ao livro razão da Contabilidade, para registrar as transações realizadas por meio de redes de internet. Estas transações ocorrem de forma descentralizada, distribuída e não podem ser alteradas. O funcionamento desta tecnologia está pautado em cadeias de blocos de dados com controle de seus conteúdos por meio de hash (uma função matemática aplicada a um conjunto de dados tornando-o único, como se fosse uma "impressão digital"); controle cronológico em que a transação ocorreu (timestamp); assinatura digital; e verificação da autenticidade dos dados transacionados pelo bloco destinatário do bloco anterior Otubo (2019).

A Blockchain tem como diferencial a sua programação que conta com a criptografia dos dados, geração da função hash e com a auditoria realizada pelos mineradores ${ }^{14}$ para confirmar a veracidade e a autoria dos dados trafegados via rede. Importante ressaltar que a mineração é definida como um processo matemático computacional Rodrigues (2017). Lucena e Henriques (2016, p. 2) apresentam os mecanismos chaves que sustentam esta tecnologia:

Seu funcionamento se baseia nos seguintes princípios: funções de mão única (normalmente funções hash), registro do tempo da criação ou modificação do arquivo (timestamp), assinatura digital do autor da alteração do arquivo, rede descentralizada peer-to-peer e mecanismo de geração de um novo bloco do blockchain.

O uso dos Smart Contracts intensificará os estudos visando a criação de um identificador único do paciente, cuja sigla em inglês é PII (personally identifiable information) ou UPID (unique patient identifier). De acordo com Mayer, Costa e Righi (2020, p. 1283) "PII é um padrão que atribui um código de identificação alfanumérico que é projetado para representar exclusivamente um paciente em um hospital"15 (tradução nossa). Por meio do acesso ao PII tanto o paciente quanto os sistemas e subsistemas dos serviços de saúde terão acesso aos seus dados. Esta iniciativa contribui para a evolução das discussões sobre a institucionalização de um PEP único e universal (contendo o histórico das consultas, exames, anamneses e procedimentos médicos); uma curadoria de PEP compartilhada com o paciente, seus responsáveis legais e com os serviços de saúde; e a permissão de acesso ao PEP ser franqueada pelo paciente às instituições de saúde e centros de pesquisa de sua confiança.

A arquitetura da Blockchain fornece à governança dos PEPs maior segurança no controle, acesso e compartilhamento de seus dados por possuir uma estrutura descentralizada de armazenamento e auditoria de suas redes. Esta estrutura é diferente da maioria utilizada para curadoria dos PEPs que são mantidos por provedores de saúde centralizados, estando as informações pessoais neles contidas vulneráveis a acessos indevidos e adulterações.

Os PEPs deverão ser avaliados quanto ao seu nível de maturidade em gestão arquivística de documentos. O termo nível de maturidade foi cunhado por Nolan (1973) para propor quatro estágios de crescimento do departamento de TI Proença, Vieira, e Borbinha (2018) e, ao longo dos anos, evoluiu em termos de metodologia de desenvolvimento Becker, Knackstedt, e Pöppelbuß (2009). O método de avaliação periódica do nível de maturidade em governança de informações foi definido por Gartner it Glossary (2021) como:

a especificação de direitos de decisão e uma estrutura de responsabilidade para garantir um comportamento apropriado na avaliação, criação, armazenamento, uso, arquivamento e exclusão de

\footnotetext{
${ }^{12}$ A Resolução Conarq n. 25, de 27 de abril de 2007, dispõe sobre a adoção do Modelo de Requisitos para Sistemas Informatizados de Gestão Arquivística de Documentos - e-ARQ Brasil pelos órgãos e entidades integrantes do Sistema Nacional de Arquivos SINAR.

${ }^{13}$ A Resolução Conarq n. 39, de 29 de abril de 2014, estabelece diretrizes para a implementação de repositórios arquivísticos digitais confiáveis para o arquivamento e manutenção de documentos arquivísticos digitais em suas fases corrente, intermediária e permanente, dos órgãos e entidades integrantes do Sistema Nacional de Arquivos - SINAR.

${ }^{14}$ Pessoas ou empresas que usam computadores com grande poder de processamento para autenticar cada passo da transação Otubo (2019).

${ }^{15}$ No original: "PII is a standard that assigns an alphanumeric identification code that is designed to uniquely represent a patient in a hospital."(Mayer et al., 2020, p. 1283).
} 
informações. Inclui os processos, funções e políticas, padrões e métricas que garantem o uso eficaz e eficiente das informações para permitir que uma organização atinja seus objetivos.

Na área Arquivística, o Arquivo Nacional propôs níveis de maturidade para programas de gestão de documentos como contribuição para a governança institucional Costa (2018). Tais níveis de maturidade devem ser observados pelos prestadores de serviços na área de saúde. Entende-se que, envidar esforços para a governança dos PEPs contribuirá para uma maior qualidade e rapidez na prestação de serviços ao paciente e que isto será possível aplicando-lhes os mais modernos métodos técnico-científicos de gestão arquivística de documentos e recursos tecnológicos que possibilitem a universalização do acesso aos seus dados e a integração dos diversos segmentos do ramo de saúde.

\section{MATERIAL E MÉTODO}

Esta pesquisa é de caráter bibliográfico, exploratório, descritivo, qualitativo e possui uma perspectiva ontológica construtivista no sentido de que este estudo seja revelador e exemplificador. Para Gil (2002, p. 41) a pesquisa exploratória "tem como objetivo proporcionar maior familiaridade com o problema, com vistas a torná-lo mais explícito ou a constituir hipóteses."A busca sistemática da literatura ocorreu nos meses de novembro a dezembro de 2020, em 7 (sete) bases de dados contendo artigos científicos produzidos no período de 2015 a 2020 :

\begin{tabular}{cl}
\hline $\mathbf{N}^{\mathbf{0}}$ & Nome \\
\hline 1 & Base de Dados Referencial de Artigos de Periódicos em Ciência da Informação - (BRAPCI) \\
\hline 2 & Library and Information Science Abstracts (Lisa/PROQUEST) \\
\hline 3 & Library, Information Science \& Technology Abstracts (Lista/EBESCO). \\
\hline 4 & Portal de periódicos da Coordenação de Aperfeiçoamento de Pessoal de Nível Superior (CAPES) \\
\hline 5 & Repositório Institucional do Instituto Brasileiro de Informação em Ciência e Tecnologia (RIDI) \\
\hline 6 & Scopus (Elsevier) \\
\hline 7 & Web of Science/Clarivate Analytics \\
\hline
\end{tabular}

Tabela 1. Bases de dados pesquisadas

Fonte: Elaborada pelos autores (2020).

As palavras-chave utilizadas para as pesquisas nas bases de dados da Tabela 1 estão elencadas abaixo:

\begin{tabular}{cl}
\hline $\mathbf{N}^{\mathbf{O}}$ & Palavras-chave \\
\hline 1 & blockchain and "record management" \\
\hline 2 & blockchain and "archives \& records" \\
\hline 3 & blockchain and "archival science" \\
\hline 4 & blockchain (para a BRAPCl e a RIDI) \\
\hline 5 & "smart contracts"and "record management" \\
\hline 6 & "smart contracts"and "archives \& records" \\
\hline 7 & "smart contracts"and "archival science" \\
\hline 8 & "smart contracts" (para a BRAPCI e a RIDI) \\
\hline
\end{tabular}

Tabela 2. Palavras-chave

Fonte: Elaborada pelos autores (2020).

Como resultado das pesquisas foram localizados 427 artigos científicos, sendo 339 contendo a palavra-chave "blockchain"e 88 "smart contracts"associadas às palavras-chave "archival science", "archives $\&$ records"e "record management", considerados termos técnicos da Arquivologia. O maior número de artigos científicos recuperados foi sobre "blockchain"e "record management", num total de 284 registros, seguido por "smart contracts"e "record management", num total de 79 registros.

Do total de 339 artigos científicos localizados sobre Blockchain e termos técnicos associados à Arquivologia, apenas 5 eram no idioma português. Um desses artigos constava tanto na base de dados Scopus quanto na CAPES, devido ao fato da pesquisa nesta última base de dados ter sido por assunto e, por conseguinte, ter retornado resultados da base Scopus. Por este motivo, este artigo foi contado uma única vez. Também um mesmo artigo foi revisado e publicado em anos e em locais diferentes sendo contado duas vezes. Ao final, 4 artigos científicos no idioma português foram encontrados nas bases de dados pesquisadas da Tabela 1.

As palavras-chave "blockchain"e "smart contracts"foram utilizadas individualmente nas bases BRAPCI e RIDI, após tentativas frustradas de recuperação de artigos científicos com os outros termos associados, sendo que, a palavra-chave "Blockchain", da Tabela 2, foi a única que propiciou retorno à pesquisa. O Google Acadêmico e outros sites de busca foram acrescidos posteriormente às buscas para complementar as pesquisas exploratórias a artigos contendo as palavras-chave "tabela de temporalidade", "prazos de guarda", "eliminação de documentos"e seus correspondentes na língua inglesa "temporality table", "custody deadlines"e "elimination 


\begin{tabular}{|c|c|c|c|c|}
\hline $\mathbf{N}^{\circ}$ & Título & Autor & Local de publicação & Palavras-chave \\
\hline 1 & $\begin{array}{l}{ }^{a} \text { Blockchain para } \\
\text { gerenciamento } \\
\text { de prontuários } \\
\text { eletrônicos. }\end{array}$ & $\begin{array}{l}\text { Viana, } \text { Caroline; } \\
\text { Brandão, Alexan- } \\
\text { dre; Dias, Diego; } \\
\text { Castellano, Gabri- } \\
\text { ela; Guimarães, } \\
\text { Marcelo. }\end{array}$ & $\begin{array}{l}\text { Revista Ibérica de Siste- } \\
\text { mas e Tecnologias de In- } \\
\text { formação, Apr 2020, Issue } \\
\text { E28, p.177-187 }\end{array}$ & $\begin{array}{l}\text { Data Management } \\
\text { Rehabilitation } \\
\text { Medical Records } \\
\text { Government Regulations } \\
\text { Cryptography } \\
\text { Blockchain } \\
\text { Descentralização } \\
\text { Ethereum } \\
\text { Registros médicos } \\
\text { Contratos inteligentes }\end{array}$ \\
\hline 2 & $\begin{array}{l}{ }^{b} \text { Modelo de utiliza- } \\
\text { ção de tecnologia } \\
\text { Blockchain no Sis- } \\
\text { tema Registral e } \\
\text { Notarial Brasileiro. }\end{array}$ & Sasaki, Elton Eiji & $\begin{array}{l}\text { Seminário Nacional de } \\
\text { Gestão da Informação e } \\
\text { do Conhecimento, v. 1, } \\
\text { 2017. (Consórcio Mestral } \\
\text { da Rede Gic). } \\
\text { Congresso de Gestão Es- } \\
\text { tratégica da Informação, } \\
\text { Empreendedorismo e Ino- } \\
\text { vação, v. 2, 2019. (Consór- } \\
\text { cio Mestral da Rede Gic). }\end{array}$ & $\begin{array}{l}\text { Blockchain. } \\
\text { Autenticação. } \\
\text { Sistema Registral e Nota- } \\
\text { rial. }\end{array}$ \\
\hline 4 & $\begin{array}{l}\text { Recomendações da } \\
\text { Diplomática para o } \\
\text { uso de Documen- } \\
\text { tos Arquivísticos Di- } \\
\text { gitais nas platafor- } \\
\text { mas do tipo Block- } \\
\text { chain. }\end{array}$ & $\begin{array}{l}\text { Nascimento, } \\
\text { Cynthia Giova- } \\
\text { nia Fernandes do; } \\
\text { Dorneles, Sander- } \\
\text { son Lopes. }\end{array}$ & $\begin{array}{l}\text { Archeion Online, n. 2, v. 7, } \\
\text { p. 26-42, 2020. (Artigo de } \\
\text { Revisão) }\end{array}$ & Não possui \\
\hline
\end{tabular}

Tabela 3. Artigos científicos encontrados no idioma português nas bases de dados pesquisadas Fonte: Elaborada pelos autores (2020).

${ }^{a}$ Este artigo foi localizado nas bases de dados SCOPUS e CAPES.

${ }^{b}$ Este artigo foi contado duas vezes por ter sido revisado e apresentado em locais e anos diferentes.

of documents"associados às tecnologias Blockchain e Smart Contracts. O único artigo científico localizado que atendeu às expectativas foi Provable and Traceable Assured Deletion (PTAD) ${ }^{16}$, dos autores Mengyu, Zhang, Yang, e Shen (2019). Os documentos publicados pela CTDE do Conarq, em especial o e-Arq-Brasil e o Glossário de Documentos Arquivísticos Digital, foram revisitados para complementar os estudos.

\section{RESULTADOS E DISCUSSÕES}

O tratamento dos PEPs é o alicerce para iniciativas de implantação de serviços médicos inteligentes e requer o estabelecimento de uma Arquitetura da Informação capaz de estruturar as informações em seus diversos ambientes organizacionais. Duque e Lyra (2011, p. 44-45) indagam "Como fazer uso eficaz dos recursos de TI sem pensar antes em uma arquitetura da informação?". Uma arquitetura da informação adequada aos PEPs demanda soluções arquivísticas e tecnológicas capazes de propiciar a manutenção das suas características de relação orgânica, unicidade, confiabilidade, autenticidade e acessibilidade (Conselho Nacional de Arquivos, 2020b, p. 36-38).

Durante os estudos sobre os PEPs identificou-se a existência de prazos de guarda e destinação distintos para prontuários em geral e de servidores públicos, bem como o tratamento dessa matéria em dois normativos, sendo o primeiro a Lei $\mathrm{n}^{\circ}$ 13.787, de 2018, e o segundo a Portaria AN n. 47, de 14 de fevereiro de 2020. A uniformização da temporalidade e destinação dos PEPs no Brasil se faz necessária, sendo oportuno o tratamento dos prazos de guarda e destinação dos PEPs de servidores públicos na respectiva lei.

As tecnologias Blockchain e Smart Contracts possuem vantagens e desvantagens, como toda tecnologia emergente, sendo necessário um estudo prévio do contexto antes de se optar pela sua utilização. As blockchains públicas ${ }^{17}$ oferecem riscos de segurança devido à impossibilidade de se exercer controle sobre acessos e operações em suas

\footnotetext{
${ }^{16}$ Exclusão garantida provável e rastreável (PTAD) (tradução nossa).

17 Blockchain pública "permite que qualquer interessado ingresse na rede e seja participante, editor e validador de novos blocos. A desvantagem desse tipo de rede é que todas atividades e informações básicas, incluindo remente e destinatário, são públicos e transparentes (2020, p. 41).
} 
redes, motivo pelo qual é sugerido o uso de blockchains privadas ${ }^{18}$ e permissionadas, "nas quais a governança é de responsabilidade de um consórcio de corpos"Lemieux, Flores, e Lacombe (2018) para a gestão tecnológica dos PEPs.

O emprego da tecnologia Blockchain possui algumas objeções para a gestão de PEPs, sendo uma delas a necessidade de eliminação (exclusão/deleção) de documentos em sistemas de gestão arquivística de documentos ou de negócios de acordo com a sua temporalidade. O motivo é que uma das características da tecnologia Blockchain é a imutabilidade de seus registros. Esta é uma das razões pelas quais os Smart Contracts são recomendados para a gestão dos PEPs, ou seja, por permitir a exclusão de registros. Uma das possíveis formas de eliminação de documentos dos PEPs, considerados sem valor para a guarda permanente, foi encontrada no artigo científico que trata da exclusão de registros nas nuvens chamado Provable and Traceable Assured Deletion (PTAD) cuja tradução é Exclusão Garantida Provável e Rastreável (PTAD), dos autores Mengyu et al. (2019).

Segundo os autores, este método permite a exclusão de registros incluídos em redes Blockchain e garante que os dados excluídos sejam irrecuperáveis. O diferencial da solução PTAD é que ela opera em três estágios desvinculação, sobrescrita dos dados e verificação da transação efetuada Mengyu et al. (2019). Esta verificação ocorre por meio dos Smart Contracts conforme explicam os criadores dessa solução "utilizamos a técnica de contrato inteligente em blockchain para executar automaticamente a verificação e manter a transação no livro razão para rastreamento"(tradução nossa) ${ }^{19}$ (Mengyu et al., 2019, p. 1). Para legitimidade e validação da PTAD, os autores propõem uma rede de consórcios composta por autoridades reguladoras e agências de avaliação para supervisionarem a exclusão garantida Mengyu et al. (2019). Para eles, esta solução é diferente e mais segura que outros métodos utilizados, como por exemplo, a desvinculação, cujos dados excluídos permanecem na nuvem e podem ser recuperados.

Como a tecnologia Blockchain não preserva o arquivo digital do documento, mas somente os ledgers ${ }^{20}$ de autenticação Flores (2018), a governança dos PEPs deve contemplar as Diretrizes para a implementação de Repositórios Digitais Confiáveis de Documentos Arquivísticos para a manutenção e preservação digital dos prontuários. A gestão de PEPs em sistemas centralizados também está sujeita a riscos, tais como: atuação de hackers, erro, descuido, desinformação ou má fé de usuários autorizados a operar os sistemas, bem como problemas operacionais e obsolescência de software, hardware e mídias, uma vez que nenhuma tecnologia é infalível.

Por conta disto, entende-se que as tecnologias Blockchain e Smart Contracts junto aos repositórios digitais confiáveis podem contribuir para proteger os PEPs contra acessos indevidos, adulterações e perdas de dados, uma vez que as mesmas informações e operações referentes aos prontuários são replicadas em diversos computadores, chamados de nós, e auditadas pelos mineradores (computadores) quanto à sua autenticidade e proveniência.

As organizações devem adotar três estratégias para obterem êxito em projetos de governança dos PEPs, em apoio aos empreendimentos na área de Saúde 4.0: instituir a gestão arquivística dos PEPs; implantar sistema de gerenciamento desses PEPs baseado nas tecnologias Blockchain e Smart Contracts; e acrescentar a obrigatoriedade da existência de Programa de Gestão Arquivística de PEPs na lista de exigências para acreditação ${ }^{21}$ dos serviços de saúde. Os níveis de maturidade em gestão arquivística de documentos a serem estipulados para verificação nas acreditações devem ser avaliados periodicamente. A pesquisa sistemática sobre as tecnologias Blockchain e Smart Contracts associadas a programas de gestão arquivística de documentos resultou um pequeno número de artigos científicos produzidos no cenário nacional. Por este motivo, o fomento de novas pesquisas em programas de pós-graduação é necessário para o aprofundamento das questões arquivísticas e tecnológicas envolvendo a governança e a curadoria dos PEPs para benefício de toda a área de saúde e, principalmente, de seus pacientes.

\section{CONSIDERAÇÕES FINAIS}

O tema desta pesquisa foi escolhido pelo fato de as tecnologias Blockchain e Smart Contracts serem consideradas inovadoras, disruptivas e promissoras para o atual e futuro cenário mundial. A área de Saúde 4.0 será uma das que mais se desenvolverá nos próximos anos, impulsionada pela pandemia da Covid-19, iniciada em 2019. A confiança em uma governança adequada dos PEPs será propulsora para a inovação em produtos e serviços. Muitos resultados poderão ser comprometidos ou frustrados sem uma gestão arquivística eficiente destes prontuários.

As pesquisas na área de Arquitetura da Informação com foco nas contribuições da Arquivística e das tecnologias Blockchain e Smart Contracts para a governança e curadoria dos PEPs são escassas, fato que levou à exploração

\footnotetext{
${ }^{18}$ Blockchain privada: "[...] surgiu de uma demanda de controle de acesso às atividades realizadas dentro da rede. Nesse caso, apenas nós autorizados podem ingressar e realizar ações permitidas."(2020, p. 41).

${ }^{19}$ No original: "We utilize technique of smart contract in blockchain to automatically execute verification and keep transaction in ledger for tracking"(Mengyu et al., 2019, p. 1)

${ }^{20}$ Ledgers: significa livro razão.

${ }^{21}$ As instituições que mais fornecem acreditações no Brasil são a Organização Nacional de Acreditação (ONA) e a Joint Comission Internacional (JCI) cujas atuações contribuem, não somente, para elevar os níveis de satisfação na prestação dos serviços médicos, mas também, para estabelecer metodologias e protocolos de segurança a nível mundial.
} 
deste assunto no sentido de contribuir cientificamente com essas áreas. O artigo científico "Provable and Traceable Assured Deletion (PTAD)", dos autores Mengyu et al. (2019), foi elucidativo em demonstrar a possibilidade de exclusão de dados em nuvem, baseados em Blockchain, e do emprego de Smart Contracts para executar, automaticamente, a verificação da exclusão e manter esta transação de exclusão no livro razão para rastreamento. Esta metodologia deverá ser objeto de pesquisas aplicadas aos PEPs, operados em Blockchain e Smart Contracts, para prova de conceito em funcionalidades de controle de prazos e de eliminação de PEPs, de acordo com a temporalidade dos documentos.

A evolução da gestão dos PEPs poderá contribuir para a emancipação dos direitos do proprietário dos dados pessoais de saúde, que é o paciente, de uma forma disruptiva e inovadora assim como são as tecnologias Blockchain e Smart Contracts. Os PEPs poderão ser compartilhados pelo paciente e pelas operadoras de saúde por meio do estabelecimento de códigos abertos e padrões de interoperabilidade com a segurança da Blockchain. Os Smart Contracts poderão otimizar a gestão dos PEPs por meio da programação de sistemas contendo algoritmos com comandos autoexecutáveis bem como da associação de inteligência artificial e robótica para interação direta com o paciente e operadoras de serviços de saúde. A gestão arquivística poderá agregar valor à toda evolução tecnológica dos PEPs por meio de uma curadoria dos documentos desde a sua produção até a sua destinação final tornando o seu acesso ágil, seguro e sem duplicidade. O monitoramento dos prazos de guarda e destinação dos PEPs poderão garantir uma base de dados idônea e atualizada para servir não somente, aos pacientes, mas à pesquisa em prol dos avanços da Saúde em benefício da atual e futuras gerações. Se tudo isto ocorrer, poderá surgir o paciente 4.0 que será aquele altamente tecnológico, independente, detentor e controlador do direito de acesso e manutenção de seus dados pessoais de saúde. 


\section{REFERÊNCIAS}

Araújo, C. A. Á. (2018). O que é Ciência da Informação? Belo Horizonte, MG: KMA.

Becker, J., Knackstedt, R., \& Pöppelbuß, J. (2009). Developing maturity models for it management. Business 8 Information Systems Engineering (BISE), 1(3), 213-222. doi: 10.1007/s12599-009-0044-5

Braga, M. M. G., \& Roncaglio, C. (2019). Os usos do termo diagnóstico na literatura arquivística. Em Questão, 25(1), 390-413. doi: 10.19132/1808-5245251.390-413

Castells, M. (2013). A sociedade em rede. São Paulo: Paz e Terra. (Vol. 1)

Cedro, L. F. d. A. (2020). Tecnologia blockchain como auxílio para transparência dos resultados de ensaios clínicos [Dissertação de Mestrado]. Universidade de Brasília.

Conselho Nacional de Arquivos. (2020a). Glossário documentos arquivísticos digitais. How it was published. Rio de Janeiro. Recuperado de https://www.gov.br/ conarq/pt-br/assuntos/camaras-tecnicas-setoriais-inativas/ camara-tecnica-de-documentos-eletronicos-ctde/ glosctde_2020_08_07.pdf

Conselho Nacional de Arquivos. (2020b). Modelo de requisitos para sistemas informatizados de gestão arquivistica de documentos - e-arq brasil. How it was published. Rio de Janeiro. Recuperado de https://www.gov.br/conarq/pt-br/ assuntos/noticias/conarq-abre-consulta-publica-visando-a -atualizacao-do-e-arq-brasil/EARQ_v2_2020_final.pdf

Costa, L. C. (2018). Contribuições para a governança institucional. In Seminário do siga, 6 (v. 28, p. 1-14). Brasília: Arquivo Nacional. Recuperado de http://siga.arquivonacional.gov.br/images/ siga_novo/SEMINARIOS/VI_SEMINARIO_SIGA/ APRESENTACOES/01-LARISSA---Programa-de-Gesto -de-Documentos.pdf (28 slides)

Decreto n. 10.148, de 2 de dezembro de 2019. (2019). Institui a comissão de coordenação do sistema de gestão de documentos e arquivos da administração pública federal, dispõe sobre a comissão permanente de avaliação de documentos, as subcomissões de coordenação do sistema de gestão de documentos e arquivos da administração pública federal $e$ o conselho nacional de arquivos, e dá outras providências. Brasília, DF: Presidência da República. Recuperado de http://www.planalto.gov.br/ccivil_03/_ato2019-2022/ 2019/decreto/D10148.htm

Drescher, D. (2018). Blockchain básico: uma introdução não técnica em 25 passos. São Paulo: Novatec; Apress. (Vol. 1)

Duque, C. G., \& Lyra, M. R. (2011). O posicionamento da Arquitetura da Informação na governança de TI. Brazilian Journal of Information Science: research trends, 4(2), 41-46. doi: 10.36311/1981-1640.2010.v4n2.01.p41

Ethereum. (2021). Introduction to smart contracts. Recuperado de https://ethereum.org/en/developers/docs/smart -contracts/\#what-is-a-smart-contract

Ferraz, R. N. (2019). As tecnologias envolvendo os contratos inteligentes (smart contracts) e alguns dos impactos nos contratos. Recuperado de https://repositorio.ufpe.br/bitstream/123456789/37502/ 1/TCC_RobersonNovellinoFerraz_51018543449_31_10 2019.pdf

Flores, D. (2018). Transformação digital por inovação sustentada ou tecnologias disruptivas em arquivos. Re- cuperado de https://www.researchgate.net/publication/ 331284400 TRANSFORMACAO DIGITAL POR INOVACĀO SUSTENTADA OU TECNÖLOGIAS DISRUPTIVAS_EM_ARQUIVOS_Daniel_Flores Programa_de_Pos-Graduacao_em_Cienciā_da Informacaō_Instituto_de_Arte_e_Comunicacao Social_Universidad

Gartner it Glossary. (2021). Business Intelligence (BI). Recuperado de https://www.gartner.com/en/information -technology/glossary/information-governance

Gil, A. C. (2002). Como elaborar projetos de pesquisa (4a. ed.). São Paulo: Atlas.

Gonçalves, A. (2006). O conceito de governança. In Congresso nacional do conpedi/uea (v. 28, p. 1-14). Manaus, AM, Brasil: Arquivo Nacional. Recuperado de https://egov.ufsc.br/portal/categoria/tags/defini\%C3\% A7\% C3\%B5es (15)

Jardim, J. M. (2018). Governança arquivística: contornos para uma noção. Acervo, 31(3), 31-45. Recuperado de http://revista.arquivonacional.gov.br/index.php/ revistaacervo/article/view/987/994

Lei n. 13.787, de 27 de dezembro de 2018. (2018). Dispõe sobre a digitalização e a utilização de sistemas informatizados para a guarda, o armazenamento e o manuseio de prontuário de paciente. Presidência da República. Recuperado de http://www.planalto.gov.br/ccivil_03/_ato2015-2018/ 2018/lei/L13787.htm

Lemieux, V., Flores, D., \& Lacombe, C. (2018). Registro de transações imobiliárias em blockchain no brasil (rcplac-01): estudo de caso 1.

doi: $10.13140 /$ RG.2.2.16022.45123

Lopes, L. C. (2009). A nova arquivística na modernização administrativa (2a. ed.). Brasília: Projecto Editorial.

Lucena, A. U. d., \& Henriques, M. A. A. (2016). Estudo de arquiteturas dos blockchains de bitcoin e ethereum. In Encontro de alunos e docentes do dca/feec. Campinas, São Paulo: Unicamp. Recuperado de https://www.sps.fee.unicamp.br/sites/ default/files/departamentos/dca/eadca/eadcaix/artigos/ lucena_henriques.pdf (9)

Mayer, A. H., da Costa, C. A., \& Righi, R. d. R. (2020). Electronic health records in a blockchain: a systematic review. Health Informatics Journal, 26(2), 1273-1288. doi: $10.1177 / 1460458219866350$

Mengyu, Z., Zhang, H., Yang, Y., \& Shen, Q. (2019, jun). Ptad: provable and traceable assured deletion in cloud storage. In Ieee symposium on computers and communications. Barcelona, Espanha: IEEE. Recuperado de https://www .sps.fee.unicamp.br/sites/default/files/departamentos/ dca/eadca/eadcaix/artigos/lucena_henriques.pdf doi: 10.1109/ISCC47284.2019.8969763

Nabeto, A. M. S. (2020). A transformação digital no sector da saúde [Dissertação de Mestrado]. Instituto Superior de Gestão. Recuperado de https://comum.rcaap.pt/bitstream/ 10400.26/33074/1/Tese\%20Mestrado\%20Ana\%20Nabeto\% 2030Junho\%202020.pdf

Nakamoto, S. (2009). Bitcoin: a peer-to-peer electronic cash system. Recuperado de https://bitcoin.org/bitcoin.pdf

Nolan, R. L. (1973). Managing the computer resource: a stage hypothesis. Communications of the ACM, 16(7), 399-405. Recuperado de https://dl.acm.org/doi/10.1145/ 362280.362284 
Otubo, F. (2019). Decifrando o blockchain. entrevistado: Marcos antônio simplício júnior. Revista Pesquisa Fapesp, 20(278), 72-75. Recuperado de https://revistapesquisa .fapesp.br/decifrando-o-blockchain/

Portaria AN n. 47, de 14 de fevereiro de 2020. (2020). PDispõe sobre o Código de Classificação e Tabela de Temporalidade e Destinação de Documentos relativos às atividadesmeio do Poder Executivo Federal. Rio de Janeiro, RJ: Ministério da Justiça e Segurança Pública. Recuperado de https://www.in.gov.br/web/dou/-/portaria-n-47-de-14 -de-fevereiro-de-2020-244298005

Proença, D., Vieira, R., \& Borbinha, J. (2018, oct). Avaliação de maturidade da governança da informação em arquivos. In Congresso nacional bad. Lisboa, Portugal. Recuperado de https://www.bad.pt/publicacoes/index.php/ congressosbad/article/view/1709/pdf

Resolução CFM n. 1.638, de 10 de julho de 2002. (2002). Define prontuário médico e torna obrigatória a criação da Comissão de Revisão de Prontuários nas instituições de saúde. Brasília: Ministério da Justiça e Segurança Pública. Recuperado de https://sistemas.cfm.org.br/normas/ visualizar/resolucoes/BR/2002/1638

Resolução Conarq n. 25, de 27 de abril de 2007. (2007). Dispõe sobre a adoção do Modelo de Requisitos para Sistemas Informatizados de Gestão Arquivistica de Documentos - e-ARQ Brasil pelos órgãos e entidades integrantes do Sistema Nacional de Arquivos - SINAR. Recuperado de https://www.gov.br/ conarq/pt-br/legislacao-arquivistica/resolucoes-do-conarq/ resolucao-no-25-de-27-de-abril-de-2007

Resolução Conarq n. 39, de 29 de abril de 2014. (2014). Estabelece diretrizes para a implementação de repositórios arquivísticos digitais confiáveis para o arquivamento $e$ manutenção de documentos arquivísticos digitais em suas fases corrente, intermediária e permanente, dos órgãos e entidades integrantes do Sistema Nacional de Arquivos - SINAR. Recuperado de https://www.gov.br/ conarq/pt-br/legislacao-arquivistica/resolucoes-do-conarq/ resolucao-no-39-de-29-de-abril-de-2014

Rodrigues, C. K. d. S. (2017, jul./dez.). Uma análise simples de eficiência e segurança da tecnologia blockchain. Revista de Sistemas e Computação-RSC, 7(2). Recuperado de https:// revistas.unifacs.br/index.php/rsc/article/view/5078

Viana, C., Brandão, A., Dias, D. R. C., Castellano, G., \& Guimarães, M. d. P. (2020). Blockchain para gerenciamento de prontuários eletrônicos. Revista ibérica de sistemas e tecnologias de informação, 1(28). Recuperado de https:// www.researchgate.net/publication/339795838_Blockchain _para_gerenciamento_de_prontuarios_eletronicos

Vidotti, S. A. B. G., Cusin, C. A., \& Corradi, J. A. M. (2008). Acessibilidade digital sob o prisma da Arquitetura da Informação. In J. A. C. Guimarães \& M. S. L. Fujita (Eds.), Ensino e pesquisa em Biblioteconomia no Brasil: a emergência de um novo olhar. São Paulo: Cultura Acadêmica.

Como citar este artigo (APA):

Xavier, A. C. \& Gottschalg-Duque, C. (2021). Prontuário eletrônico do paciente: qual a contribuição da arquivística e do Smart Contracts para a sua gestão na Era da Saúde 4.0?. AtoZ: novas práticas em informação e conhecimento, 10(3), 1 - 10. Recuperado de: http://dx.doi.org/10.5380/atoz.v10i3.81267 